\begin{tabular}{ll}
\hline \hline MINING AND METALLURGY INSTITUTE BOR & ISSN: 2334-8836 (Štampano izdanje) \\
UDK: 622 & ISSN: 2406-1395 (Online) \\
\hline \hline
\end{tabular}

UDK: $622.271 / .33(045)=111$

doi:10.5937/mmeb1702023S

Nikola Stanić* Saša Stepanović, Dejan Bugarin", Miljan Gomilanović*

\title{
SELECTION THE RATIONAL MODEL OF \\ TRANSPORT TRUCK BY THE SELECTIVE COAL MINING AT THE OPEN PIT GACKO**
}

\begin{abstract}
This paper gives the selection of the optimal model of trucks for transport of coal, and seam waste at the OP Gacko in terms of selective exploitation. Mining of coal and seam waste is carried out in two exploitation zones, the central and roof zone, and the coal and waste transport and disposal is carried out to the different crushing plants and landfills. Using the software package Talpak, the capacity of a truck for transport of coal, and seam waste was calculated for different transport routes and types of trucks. The analysis was done for three types of trucks: Belaz 7555, Belaz 75491 and Belaz.75135. The results analysis were used for evaluation, ranking and selection the optimal type of truck.
\end{abstract}

Keywords: truck transport, OP Gacko, transportation costs

\section{INTRODUCTION}

Works on coal mining at the open pit Gacko currently performed by the Main mining project of the open pit Gacko - Central field capacity of $2.3 \cdot 10^{6} \mathrm{t} /$ year of runof-mine coal. Mining takes place in two zones, the roof and central exploitation zone.

Excavation the overburden and interseam waste in the central zone of the open pit (the zone of exploitation the main and the first and the second floor of coal seam), is carried out within the second continuous BTO system and within the DTO the combined system. Overburden excavated by the bucket wheel excavator type ER 1250 $17 / 1.5$ within the first BTO system is transported by conveyor belts with a belt width of $1200 \mathrm{~mm}$ and disposed on the West outer landfill by a spreader Ars $1200(20+50) *$ 21 . In the case of the second BTO system, the base of excavation equipment includes a bucket-wheel excavator ER 1250*16/1, which operates in tandem with a selfpropelled transporter P of $1600 * 21 / 50$. Disposal is done in the excavated space of the Field B. In addition to a continuous system in the excavation of overburden, the hydraulic shovel excavators Komatsu PC 2000, Terex RH 90C, D 475 bulldozer Comatsu in tandem with a loader Komatsu WA 700 and a combine Wirtgen 2500SM. Transport of overburden is carried out by trucks, capacity of $110 \mathrm{t}$ (trucks BELAZ 75135) to the crusher SB are 1525 (DTO system).

\footnotetext{
* Mining and Metallurgy Institute Bor, e-mail: nikola.stanic@irmbor.co.rs

** This work is derived from the project TR37001 "The Impact of Mining Waste from RTB Bor on the Pollution of Surrounding Water Systems with the Proposal of Measures and Procedures for Reduction Harmful Effect on the Environment", funded by the Ministry of Education, Science and Technological Development of the Republic of Serbia
} 


\section{TRANSPORTATION SYSTEMS OF COAL AND SEAM WASTE}

Coal mining is done applying the technology of selective mining. The following hydraulic shower excavators are engaged on these activities are engaged: Komatsu PC 800, Hyundai R800, and CAT 385B. Removed quantities of coal are transported by truck $55 \mathrm{t}$ (BELAZ 7555) to the primary crusher for coal SB 1315, and SB 1515 (DTD system).
Transportation of coal and waste excavated in the process of selective coal mining is divided into internal and external. Having in mind, the coal excavation and seam waste takes place in two spatially separate exploitation wholes, the central zone and roof zone. In each zone and for different periods of development work, there are the appropriate schemes of coal and seam waste transport, given in Figures 1 and 2.

\section{CENTRAL EXPLOITATION ZONE}

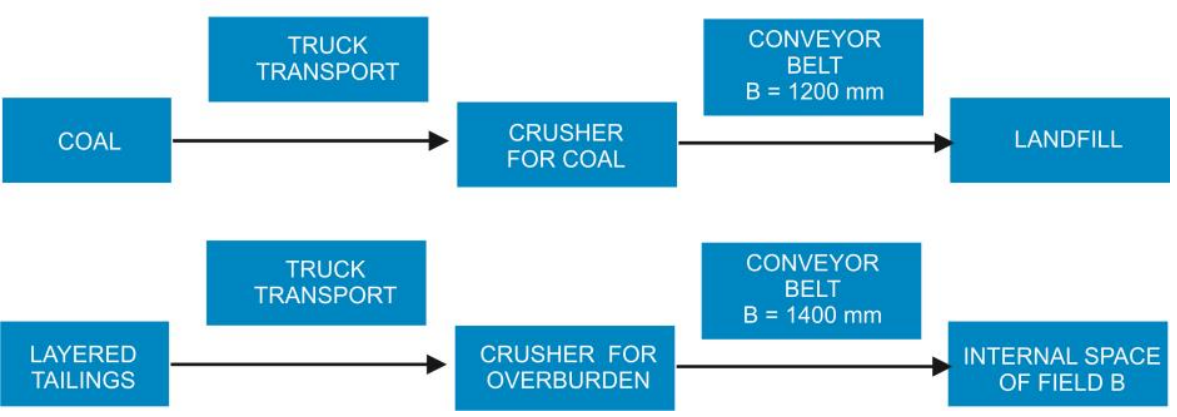

Figure 1 Scheme of coal and waste transport in the central zone of exploitation

\section{ROFF ZONE OF EXPLOITATION}
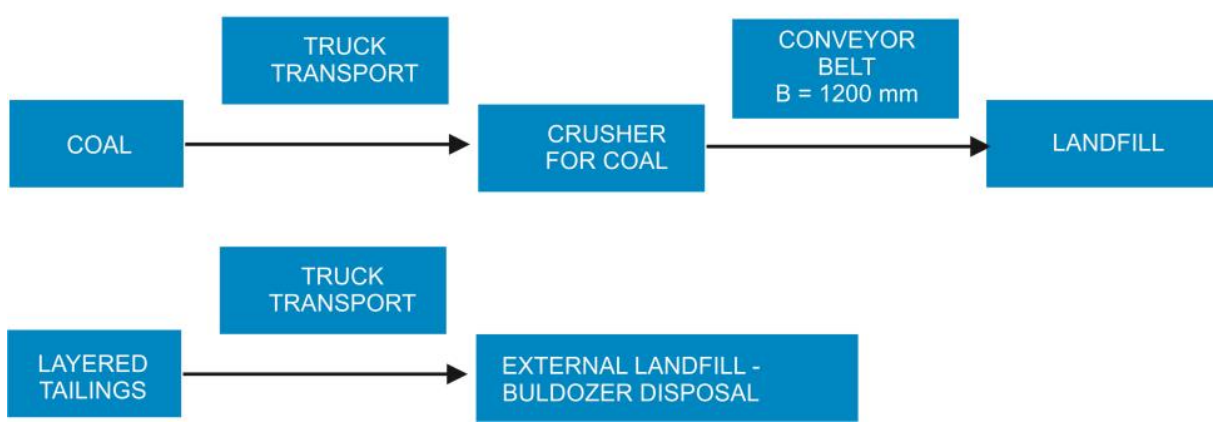

Figure 2 Scheme of coal and waste in the roof zone of exploitation 


\section{CALCULATION THE TRUCK TRANSPORT}

Internal transport of coal is by trucks and takes place from the excavation site to the crushing plants for coal.

Internal transport of waste is by truck in the central zone of exploitation and takes place from the excavation site to the crushing plant for waste, or DTO crusher system. Transport of waste in the roof zone of exploitation is by truck from the excavation site to a place of disposal on the external landfill.

External transport of coal takes place from the crushing plants to the landfill of the power plant by belt conveyors, belt width $1200 \mathrm{~mm}$. The external transport of waste from the central coal series takes place from the crushing plant for waste to the site of disposal in ther excavated area of the Field B. Disposal of waste tailings is carried out within a disposal of overburden from the central zone of the open pit, or by a combined system (DTO, crusher-conveyor beltstacker). Disposal is done by a stacker type OS 1600.
Computer program Talpac was used for calculation the system excavator - truck used a computer program Talpac. Based on the input parameters, a hourly capacity of the truck was calculated for a particular relation.

Software package Talpak is a simulation of the loading and transport process at the open pits. The software optimizes the transport fleet budget, calculation the technical and economic parameters of equipment operation such as a cycle length, capacity, etc. In the present case, this program was used to determine the parameters in transport of various materials (coal and waste) and from different sites (the central zone and roof zone of exploitation). The calculation results are used as the base for the operating costs of transport.

The organization of work at the open pit Gacko is 365 days a year, 7 days a week in 3 shifts. Total effective time during the year amounted to 3,500. Based on these organizations, following the planned working time was used for calculation the loading and transport:
- Total possible number of shifts per year:

- Duration of a shift:

- Operating hours per year:

- Effective working hours per shift

- Effective working hours per year

Presented planned and effective working hours at the open pit was used in the software Talpac for calculation the loading and transport system. Table 1 shows the input

\author{
$1,095 \mathrm{shift} /$ year \\ $8 \mathrm{~h}$ \\ 8,760 hours \\ $5.5 \mathrm{~h}$ \\ 3,500 hours
}

parameters and loading mechanization used for calculation. Table 2 shows the input parameters for the transport mechanization required for calculation. 
Table 1 Characteristics of loading machinery

\begin{tabular}{|l|l|l|l|c|}
\hline Komatsu PC 800 & \multicolumn{5}{|c|}{ Parameters } \\
\cline { 2 - 5 } & Showel volume $\left(\mathrm{m}^{3}\right)$ & 6 & Motor power $(\mathrm{kW})$ & 363 \\
\hline Speed $(\mathrm{km} / \mathrm{h})$ & 4.2 & No. of revolutions $(\mathrm{rpm})$ & 1800 \\
\hline Excavation depth $(\mathrm{m})$ & 8.45 & Weight $(\mathrm{kg})$ & 75200 \\
\hline $\begin{array}{l}\text { Medium ground } \\
\text { pressure }\left(\mathrm{N} / \mathrm{cm}^{2}\right)\end{array}$ & 12.2 & Length of rig (mm) & 7500 \\
\hline $\begin{array}{l}\text { Caterpillar width } \\
(\mathrm{mm})\end{array}$ & 610 & Total length (mm) & 14405 \\
\hline $\begin{array}{l}\text { Width of caterpillar } \\
\text { base (mm) }\end{array}$ & 4110 & Height of branch & 4690 \\
\cline { 2 - 6 } & $\begin{array}{l}\text { Length of caterpillar } \\
\text { base (mm) }\end{array}$ & 5810 & Cabin height & 3670 \\
\cline { 2 - 6 } & Shovel width (mm) & 2200 & Total width & 4110 \\
\hline
\end{tabular}

Table 2 Characteristics of transport mechanization

\begin{tabular}{|c|c|c|}
\hline \multicolumn{3}{|l|}{ Truck Belaz 7555-55 tons } \\
\hline \multirow{10}{*}{ 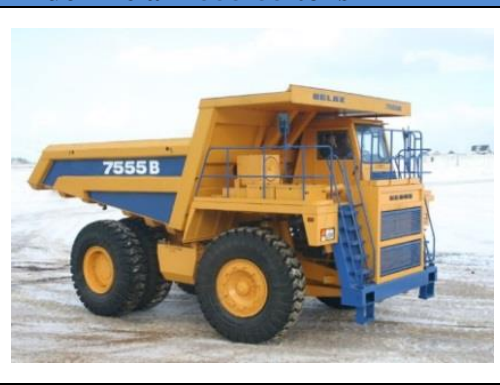 } & Hopper capacity $\left(\mathrm{m}^{3}\right)$ & 37.5 \\
\hline & Engine power $(\mathrm{kW})$ & 522 \\
\hline & Capacity $(\mathrm{t})$ & 55 \\
\hline & Max. speed (km / h) & 55 \\
\hline & Dump angle $\left({ }^{\circ}\right)$ & 47 \\
\hline & Weight $(\mathrm{kg})$ & 41000 \\
\hline & Total height $(\mathrm{mm})$ & 4610 \\
\hline & Total width $(\mathrm{mm})$ & 5240 \\
\hline & Total length (mm) & 8890 \\
\hline & Price $(\mathrm{KM})$ & 800000 \\
\hline \multicolumn{3}{|l|}{ Truck Belaz 75491-80 tons } \\
\hline \multirow{10}{*}{ (6) } & Hopper capacity $\left(\mathrm{m}^{3}\right)$ & 46 \\
\hline & Engine power $(\mathrm{kW})$ & 630 \\
\hline & Capacity (t) & 80 \\
\hline & Max. speed $(\mathrm{km} / \mathrm{h})$ & 50 \\
\hline & Dump angle $\left({ }^{\circ}\right)$ & 46 \\
\hline & Weight $(\mathrm{kg})$ & 72500 \\
\hline & Total height $(\mathrm{mm})$ & 5350 \\
\hline & Total width $(\mathrm{mm})$ & 5420 \\
\hline & Total length $(\mathrm{mm})$ & 10300 \\
\hline & Price $(\mathrm{KM})$ & 1300000 \\
\hline \multicolumn{3}{|l|}{ Truck Belaz 75135-110 tons } \\
\hline \multicolumn{3}{|l|}{1 ruck belaz /5155-110 tons } \\
\hline \multirow{9}{*}{ 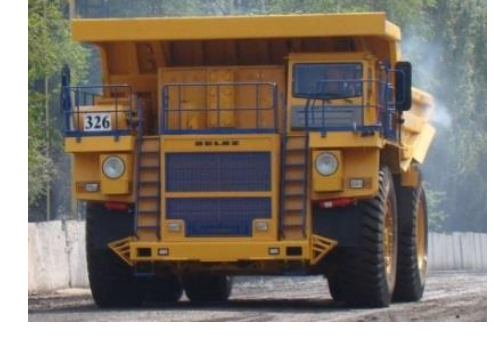 } & Engine power $(\mathrm{kW})$ & 895 \\
\hline & Capacity (t) & 110 \\
\hline & Max. speed (km / h) & 50 \\
\hline & Dump angle $\left({ }^{\circ}\right)$ & 47 \\
\hline & Weight $(\mathrm{kg})$ & 100100 \\
\hline & Total height $(\mathrm{mm})$ & 5900 \\
\hline & Total width (mm) & 6400 \\
\hline & Total length $(\mathrm{mm})$ & 11500 \\
\hline & Price $(\mathrm{KM})$ & 1800000 \\
\hline
\end{tabular}


Based on the entered data, the hourly capacities of the truck were calculated for the certain relation expressed in $\mathrm{cm}^{3} / \mathrm{h}$ when operating in conjunction with the loading equipment. Based on the amounts that should be transported into a specific route and hours of operation, for each zone of work implementation in the considered period of exploitation, the number of required trucks was calculated. Number of trucks needed in the corresponding period was calculated based on the total time required for truck engagement for the amounts of coal and seam waste from the individual work sites, and for the specific transport lengths. Tables 3, 4 and 5 are give the parameters of loading and transport system, calculated in the software package Talpac. Calculations are made according to the annual periods for the three types of trucks (Belaz 7555, Belaz 75491, Belaz 55135), depending on the zone of exploitation (roof and central zone) and the type of materials (coal and seam waste).

Table 3 Parameters of transport by the trucks type Belaz 7555

\begin{tabular}{|c|c|c|c|c|c|c|c|}
\hline 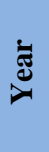 & Place & $\begin{array}{c}\text { Central } \\
\text { Zone - } \\
\text { Coal }\end{array}$ & $\begin{array}{c}\text { Central } \\
\text { Zone - } \\
\text { seam } \\
\text { waste }\end{array}$ & $\begin{array}{c}\text { Roof } \\
\text { zone - } \\
\text { coal }\end{array}$ & $\begin{array}{c}\text { Roof zone } \\
\text { - seam } \\
\text { waste }\end{array}$ & $\begin{array}{c}\text { Required } \\
\text { number } \\
\text { of trucks }\end{array}$ & $\begin{array}{c}\text { Reserve in } \\
\text { capacity } \\
(\%)\end{array}$ \\
\hline \multirow{3}{*}{ 하 } & Quantities $\left(\check{c}^{3}{ }^{3}\right)$ & 703,125 & 175,732 & $1,077,000$ & 357,712 & \multirow{3}{*}{10} & \multirow{3}{*}{$24.50 \%$} \\
\hline & $\begin{array}{l}\text { Capacity of transp. } \\
\text { truck }\left(\check{c} \mathrm{~cm}^{3} / \mathrm{h}\right)\end{array}$ & 98.8 & 89.4 & 69.9 & 47.8 & & \\
\hline & $\begin{array}{l}\text { Required time of } \\
\text { engagement }(\mathrm{h})\end{array}$ & 7,114 & 1,965 & 15,401 & 7,484 & & \\
\hline \multirow{3}{*}{$\stackrel{\infty}{\stackrel{i}{i}}$} & Quantities $\left(\check{c ̆ m}^{3}\right)$ & 703,125 & 155,418 & $1,077,000$ & 521,436 & \multirow{3}{*}{11} & \multirow{3}{*}{$19.64 \%$} \\
\hline & $\begin{array}{l}\text { Capacity of transp. } \\
\text { truck }\left(\check{\mathrm{cm}^{3}} / \mathrm{h}\right)\end{array}$ & 92.5 & 84.9 & 66.5 & 46.7 & & \\
\hline & $\begin{array}{l}\text { Required time of } \\
\text { engagement }(\mathrm{h})\end{array}$ & 7,601 & 1,831 & 16,203 & 11,156 & & \\
\hline \multirow{3}{*}{ 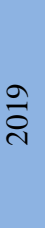 } & Quantities $\left(\mathrm{c̆m}^{3}\right)$ & 703,125 & 94,935 & $1,077,000$ & 769,835 & \multirow{3}{*}{13} & \multirow{3}{*}{$17.72 \%$} \\
\hline & $\begin{array}{l}\text { Capacity of transp. } \\
\text { truck }\left(\mathrm{c̆m}^{3} / \mathrm{h}\right)\end{array}$ & 78.3 & 68.4 & 55.54 & 46 & & \\
\hline & $\begin{array}{l}\text { Required time of } \\
\text { engagement }(\mathrm{h})\end{array}$ & 8,978 & 1,389 & 19,392 & 16,735 & & \\
\hline \multirow{3}{*}{ ત્તి } & Quantities $\left(\mathrm{cm}^{3}\right)$ & 703,125 & 208,438 & $1,077,000$ & 589,884 & \multirow{3}{*}{14} & \multirow{3}{*}{$21.51 \%$} \\
\hline & $\begin{array}{l}\text { Capacity of transp. } \\
\text { truck }\left(\check{\mathrm{cm}^{3}} / \mathrm{h}\right)\end{array}$ & 70.29 & 62.86 & 56.16 & 39.67 & & \\
\hline & $\begin{array}{l}\text { Required time of } \\
\text { engagement (h) }\end{array}$ & 10,003 & 3,316 & 19,177 & 14,870 & & \\
\hline
\end{tabular}


Table 4 Parameters of transport by trucks type Belaz 75491

\begin{tabular}{|c|c|c|c|c|c|c|c|}
\hline 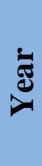 & Place & $\begin{array}{c}\text { Central } \\
\text { Zone - } \\
\text { Coal }\end{array}$ & $\begin{array}{c}\text { Central } \\
\text { Zone - } \\
\text { seam } \\
\text { waste }\end{array}$ & $\begin{array}{c}\text { Roof } \\
\text { zone - } \\
\text { coal }\end{array}$ & $\begin{array}{c}\text { Roof zone } \\
\text { - seam } \\
\text { waste }\end{array}$ & $\begin{array}{c}\text { Required } \\
\text { number } \\
\text { of trucks }\end{array}$ & $\begin{array}{c}\text { Reserve } \\
\text { in } \\
\text { capacity } \\
(\%)\end{array}$ \\
\hline \multirow{3}{*}{ 궁 } & Quantities $\left(\check{c}^{3}{ }^{3}\right)$ & 703,125 & 175,732 & $1,077,000$ & 357,712 & \multirow{3}{*}{9} & \multirow{3}{*}{$12.00 \%$} \\
\hline & $\begin{array}{l}\text { Capacity of transp. } \\
\operatorname{truck}\left(\mathrm{čm}^{3} / \mathrm{h}\right)\end{array}$ & 109.32 & 99.25 & 77.32 & 59.81 & & \\
\hline & $\begin{array}{l}\text { Required time of } \\
\text { engagement }(\mathrm{h})\end{array}$ & 6,430 & 1,771 & 13,929 & 5,981 & & \\
\hline \multirow{3}{*}{$\frac{\infty}{8}$} & Quantities ( c̆m $\left.^{3}\right)$ & 703,125 & 155,418 & $1,077,000$ & 521,436 & \multirow{3}{*}{10} & \multirow{3}{*}{$9.00 \%$} \\
\hline & $\begin{array}{l}\text { Capacity of } \\
\text { transp. truck } \\
\left(\check{c ̆ m}^{3} / \mathrm{h}\right)\end{array}$ & 96.22 & 97.03 & 72.23 & 51.99 & & \\
\hline & $\begin{array}{l}\text { Required time of } \\
\text { engagement }(\mathrm{h})\end{array}$ & 7,309 & 1,602 & 14,912 & 10,030 & & \\
\hline \multirow{3}{*}{$\frac{\text { ণิ }}{\circ}$} & Quantities $\left(\check{c}^{3}{ }^{3}\right)$ & 703,125 & 94,935 & $1,077,000$ & 769,835 & \multirow{3}{*}{11} & \multirow{3}{*}{$8.40 \%$} \\
\hline & $\begin{array}{l}\text { Capacity of } \\
\text { transp. truck } \\
\left(\check{c ̆ m}^{3} / \mathrm{h}\right)\end{array}$ & 92.84 & 89.08 & 70.53 & 58.36 & & \\
\hline & $\begin{array}{l}\text { Required time of } \\
\text { engagement }(\mathrm{h})\end{array}$ & 7,572 & 1066 & 15,270 & 13,192 & & \\
\hline \multirow{3}{*}{ ঠి } & Quantities $\left(\mathrm{cm}^{3}\right)$ & 703,125 & 208,438 & $1,077,000$ & 589,884 & \multirow{3}{*}{12} & \multirow{3}{*}{$7.80 \%$} \\
\hline & 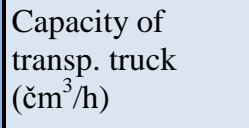 & 77.5 & 58.08 & 70.34 & 49.37 & & \\
\hline & $\begin{array}{l}\text { Required time of } \\
\text { engagement (h) }\end{array}$ & 9,072 & 3,581 & 15,312 & 11,949 & & \\
\hline
\end{tabular}


Table 5 Parameters of transport by trucks type Belaz 75135

\begin{tabular}{|c|c|c|c|c|c|c|c|}
\hline 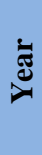 & Place & $\begin{array}{c}\text { Central } \\
\text { Zone - } \\
\text { Coal }\end{array}$ & $\begin{array}{c}\text { Central } \\
\text { Zone - } \\
\text { seam } \\
\text { waste }\end{array}$ & $\begin{array}{c}\text { Roof zone } \\
\text { - coal }\end{array}$ & $\begin{array}{l}\text { Roof zone - } \\
\text { seam waste }\end{array}$ & $\begin{array}{c}\text { Required } \\
\text { number } \\
\text { of trucks }\end{array}$ & $\begin{array}{c}\text { Reserve in } \\
\text { capacity } \\
(\%)\end{array}$ \\
\hline \multirow{3}{*}{ 호 } & Quantities (čm³) & 703,125 & 175,732 & $1,077,000$ & 357,712 & \multirow{3}{*}{6} & \multirow{3}{*}{$21.25 \%$} \\
\hline & $\begin{array}{l}\text { Capacity of transp. } \\
\text { truck }\left(\check{c}^{3} \mathrm{~m}^{3} / \mathrm{h}\right)\end{array}$ & 120.3 & 142.3 & 120.1 & 96.1 & & \\
\hline & $\begin{array}{l}\text { Required time of } \\
\text { engagement }(\mathrm{h})\end{array}$ & 5,844 & 1,235 & 8,964 & 3,721 & & \\
\hline \multirow{3}{*}{$\stackrel{\infty}{\infty}$} & Quantities $\left(\mathrm{c̆m}^{3}\right)$ & 703,125 & 155,418 & $1,077,000$ & 521,436 & \multirow{3}{*}{7} & \multirow{3}{*}{$27.48 \%$} \\
\hline & $\begin{array}{l}\text { Capacity of transp. } \\
\text { truck }\left(\check{c} \mathrm{~cm}^{3} / \mathrm{h}\right)\end{array}$ & 120.3 & 109.8 & 120.2 & 93.8 & & \\
\hline & $\begin{array}{l}\text { Required time of } \\
\text { engagement }(\mathrm{h})\end{array}$ & 5,843 & 1,415 & 8,964 & 5,559 & & \\
\hline \multirow{3}{*}{$\stackrel{\circ}{\circ}$} & Quantities (čm³) & 703,125 & 94,935 & $1,077,000$ & 769,835 & \multirow{3}{*}{7} & \multirow{3}{*}{$16.16 \%$} \\
\hline & $\begin{array}{l}\text { Capacity of transp. } \\
\text { truck }\left(\mathrm{čm}^{3} / \mathrm{h}\right)\end{array}$ & 120.2 & 109.1 & 119.9 & 90.4 & & \\
\hline & $\begin{array}{l}\text { Required time of } \\
\text { engagement }(\mathrm{h})\end{array}$ & 5,848 & 870 & 8,986 & 8,515 & & \\
\hline \multirow{3}{*}{ ત્ડ } & Quantities $\left(\mathrm{cm}^{3}\right)$ & 703,125 & 208,438 & $1,077,000$ & 589,884 & \multirow{3}{*}{7} & \multirow{3}{*}{$18.52 \%$} \\
\hline & $\begin{array}{l}\text { Capacity of transp. } \\
\text { truck }\left(\check{\mathrm{cm}}{ }^{3} / \mathrm{h}\right)\end{array}$ & 120 & 108.9 & 118.9 & 86.3 & & \\
\hline & $\begin{array}{l}\text { Required time of } \\
\text { engagement }(\mathrm{h})\end{array}$ & 5,858 & 1,915 & 9,058 & 6,835 & & \\
\hline
\end{tabular}

Based on the determined parameters of transportation the masses of coal and waste, the costs of standardized material were calculated. The standardized materials included: fuel $\left(\mathrm{kg} / \check{c}^{3}\right)$, lubricant $\left(\mathrm{kg} / \mathrm{c̆m}^{3}\right)$, an oil $\left(\mathrm{kg} / \mathrm{cm}^{3}\right)$, tires (pcs.) and spare parts $\left(\mathrm{kg} / \mathrm{cm}^{3}\right)$, Table 6 gives a summary of the standardized material costs per year for three types of trucks. Based on the calculated standardized costs, the calculation was made of the total operating costs, and they are shown in Table 7. 
Table 6 Costs of standardized material per year

\begin{tabular}{|c|c|c|c|c|c|c|c|c|c|}
\hline \multicolumn{10}{|c|}{ Normative costs per year } \\
\hline \multirow[t]{2}{*}{$\begin{array}{l}\text { Type of } \\
\text { truck }\end{array}$} & \multirow[t]{2}{*}{ Years } & $\begin{array}{l}\text { Unit costs } \\
\left(\mathbf{K M} / \mathbf{c m}^{3}\right)\end{array}$ & $\begin{array}{c}\text { Unit } \\
\text { costs } \\
(\mathrm{KM} / \mathrm{t})\end{array}$ & $\begin{array}{l}\text { Unit costs } \\
\left(\mathbf{K M} / \breve{\mathbf{c}}^{3}{ }^{3}\right)\end{array}$ & $\begin{array}{c}\text { Unit } \\
\text { costs } \\
(\mathbf{K M} / \mathbf{t})\end{array}$ & $\begin{array}{l}\text { Unit costs } \\
\left(\mathbf{K M} / \breve{c m}^{3}\right)\end{array}$ & $\begin{array}{c}\text { Unit } \\
\text { costs } \\
(\mathrm{KM} / \mathrm{t})\end{array}$ & $\begin{array}{l}\text { Unit costs } \\
\left(\mathrm{KM} / \check{c ̆ m}^{3}\right)\end{array}$ & \begin{tabular}{|c|} 
Unit \\
costs \\
$(\mathrm{KM} / \mathrm{t})$
\end{tabular} \\
\hline & & CZ-U* & CZ-U & CZ-SJ $^{\dagger}$ & CZ-SJ & KZ-U & KZ-U & KZ-SJ $^{\S}$ & KZ-SJ \\
\hline \multirow{4}{*}{$\begin{array}{l}\text { Belaz } \\
7555\end{array}$} & 2017 & 1.574 & 0.984 & 1.721 & 1.076 & 2.151 & 1.344 & 3.062 & 1.914 \\
\hline & 2018 & 1.669 & 1.043 & 1.803 & 1.127 & 2.251 & 1.407 & 3.130 & 1.956 \\
\hline & 2019 & 1.939 & 1.212 & 2.194 & 1.371 & 2.660 & 1.663 & 3.175 & 1.984 \\
\hline & 2020 & 2.140 & 1.337 & 2.371 & 1.482 & 2.633 & 1.646 & 3.652 & 2.283 \\
\hline \multirow{4}{*}{$\begin{array}{l}\text { Belaz } \\
75491\end{array}$} & 2017 & 1.685 & 1.053 & 1.838 & 1.148 & 2.308 & 1.442 & 2.931 & 1.832 \\
\hline & 2018 & 1.890 & 1.181 & 1.875 & 1.172 & 2.458 & 1.536 & 3.344 & 2.090 \\
\hline & 2019 & 1.952 & 1.220 & 2.027 & 1.267 & 2.513 & 1.570 & 2.999 & 1.874 \\
\hline & 2020 & 2.303 & 1.439 & 3.013 & 1.883 & 2.519 & 1.574 & 3.512 & 2.195 \\
\hline \multirow{4}{*}{$\begin{array}{l}\text { Belaz } \\
75135\end{array}$} & 2017 & 2.381 & 1.349 & 2.041 & 1.156 & 2.384 & 1.351 & \begin{tabular}{|l|}
2.935 \\
\end{tabular} & 1.663 \\
\hline & 2018 & 2.381 & 1.349 & 2.591 & 1.468 & 2.383 & 1.350 & 3.003 & 1.701 \\
\hline & 2019 & 2.383 & 1.350 & 2.607 & 1.477 & 2.388 & 1.353 & 3.109 & 1.761 \\
\hline & 2020 & 2.386 & 1.352 & 2.611 & 1.479 & 2.407 & 1.364 & 3.248 & 1.840 \\
\hline
\end{tabular}

Table 7 Operating costs of the different types of trucks

\begin{tabular}{|c|c|c|c|c|c|c|}
\hline & & & CZ-U & CZ-SJ & KZ-U & KZ-SJ \\
\hline \multirow{8}{*}{ 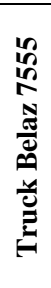 } & \multirow{2}{*}{2017} & The required quantities $\left(\check{c}^{3}{ }^{3}\right)$ & 703,125 & 175,732 & $\mathbf{1 , 0 7 7 , 0 0 0}$ & 357,712 \\
\hline & & Total (KM) & $1,106,892$ & 302,408 & $2,316,299$ & $1,095,254$ \\
\hline & \multirow{2}{*}{2018} & The required quantities $\left(\mathrm{c}^{3}{ }^{3}\right)$ & 703,125 & 155,418 & $\mathbf{1 , 0 7 7 , 0 0 0}$ & 521,436 \\
\hline & & Total (KM) & $1,173,661$ & 280,143 & $2,424,814$ & $1,631,945$ \\
\hline & \multirow{2}{*}{2019} & The required quantities $\left(\mathrm{c̆m}^{3}\right)$ & 703,125 & 94,935 & $1,077,000$ & 769,835 \\
\hline & & Total $(\mathrm{KM})$ & $1,363,556$ & 208,279 & $2,865,060$ & $2,443,918$ \\
\hline & \multirow{2}{*}{2020} & The required quantities $\left(\mathrm{c̆m}^{3}\right)$ & 703,125 & 208,438 & $\mathbf{1 , 0 7 7 , 0 0 0}$ & 589,884 \\
\hline & & Total (KM) & $1,504,519$ & 494,291 & $2,835,571$ & $2,154,515$ \\
\hline \multirow{8}{*}{ 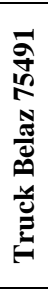 } & \multirow{2}{*}{2017} & The required quantities $\left(\mathrm{ccm}^{3}\right)$ & 703,125 & $\mathbf{1 7 5 , 7 3 2}$ & $1,077,000$ & $\mathbf{3 5 7 , 7 1 2}$ \\
\hline & & Total (KM) & $1,184,685$ & 322,920 & $2,485,396$ & $1,048,314$ \\
\hline & \multirow{2}{*}{2018} & The required quantities $\left.\left(\check{c}^{3}\right)^{3}\right)$ & 703,125 & 155,418 & $1,077,000$ & 521,436 \\
\hline & & Total $(\mathrm{KM})$ & $1,328,744$ & 291,486 & $2,646,879$ & $1,743,859$ \\
\hline & \multirow{2}{*}{2019} & The required quantities $\left.\left(\check{c}^{3}\right)^{3}\right)$ & 703,125 & 94,935 & $\mathbf{1 , 0 7 7 , 0 0 0}$ & 769,835 \\
\hline & & Total (KM) & $1,372,512$ & 192,415 & $2,706,005$ & $2,308,698$ \\
\hline & \multirow{2}{*}{2020} & The required quantities $\left(\mathrm{c̆m}^{3}\right)$ & 703,125 & 208,438 & $\mathbf{1 , 0 7 7 , 0 0 0}$ & 589,884 \\
\hline & & Total (KM) & $1,619,129$ & 627,928 & $2,712,791$ & $2,071,830$ \\
\hline \multirow{8}{*}{ 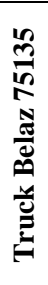 } & \multirow{2}{*}{2017} & The required quantities $\left(\check{c}^{3}{ }^{3}\right)$ & 703,125 & 175,732 & $\mathbf{1 , 0 7 7 , 0 0 0}$ & 357,712 \\
\hline & & Total $(\mathrm{KM})$ & $1,673,999$ & 358,590 & $2,568,067$ & $1,049,887$ \\
\hline & \multirow{2}{*}{2018} & The required quantities $\left(\check{c ̆ m}^{3}\right)$ & 703,125 & 155,418 & $\mathbf{1 , 0 7 7 , 0 0 0}$ & 521,436 \\
\hline & & Total $(\mathrm{KM})$ & $1,673,999$ & 402,728 & $2,566,091$ & $1,565,642$ \\
\hline & \multirow{2}{*}{2019} & The required quantities $\left(\check{c ̆ m}^{3}\right)$ & 703,125 & 94,935 & $\mathbf{1 , 0 7 7 , 0 0 0}$ & 769,835 \\
\hline & & Total $(\mathrm{KM})$ & $1,675,286$ & 247,470 & $2,572,027$ & $2,393,199$ \\
\hline & \multirow{2}{*}{2020} & The required quantities $\left(\mathrm{c}^{3}{ }^{3}\right)$ & 703,125 & 208,438 & $\mathbf{1 , 0 7 7 , 0 0 0}$ & 589,884 \\
\hline & & Total $(\mathrm{KM})$ & $1,677,867$ & 544,270 & $2,592,028$ & $1,915,858$ \\
\hline
\end{tabular}

* Central Zone - coal,

Central Zone - seam waste

Roof zone - coal,

$\$$ Roof zone - seam waste 


\section{CAPITAL AND OPERATING \\ COSTS OF VARIANT SOLUTIONS}

In order to gain a clearer status on the costs of variant solutions, the calculation of the capital costs was made. Capital expenditure (capex) were calculated as annuity loans for the purchase of a truck loan. The loan was calculated for the following conditions: the return period 10 years, interest rate

of $6 \%$. The operating costs (opex) included the total standardized costs per year in the areas of exploitation. Table 8 gives the total values of capital and operating costs. Figure 3 and Figure 4 shows a graph of movement the capital and operating costs for three types of trucks.

Table 8 Capital and operating costs (KM)

\begin{tabular}{cccccc|c|}
$\begin{array}{c}\text { Type of } \\
\text { truck }\end{array}$ & costs & $\mathbf{2 0 1 7}$ & $\mathbf{2 0 1 8}$ & $\mathbf{2 0 1 9}$ & $\mathbf{2 0 2 0}$ & \multicolumn{1}{c}{$\boldsymbol{\Sigma}$} \\
\hline \multirow{2}{*}{ Belaz 7555 } & opex & $4,820,853$ & $5,510,564$ & $6,880,814$ & $6,988,895$ & $24,201,126$ \\
& capex & $1,280,000$ & $1,360,000$ & $1,563,200$ & $1,628,800$ & $5,832,000$ \\
\hline \multirow{2}{*}{ Belaz 75491 } & opex & $5,041,315$ & $6,010,968$ & $6,579,629$ & $7,031,678$ & $24,663,590$ \\
& capex & $2,402,000$ & $2,009,800$ & $2,139,800$ & $2,262,000$ & $8,813,600$ \\
\hline \multirow{2}{*}{ Belaz 75135 } & opex & $5,650,541$ & $6,208,460$ & $6,887,982$ & $6,730,024$ & $25,477,007$ \\
& capex & $1,728,000$ & $1,951,200$ & $1,875,600$ & $2,008,000$ & $7,562,800$ \\
\hline
\end{tabular}

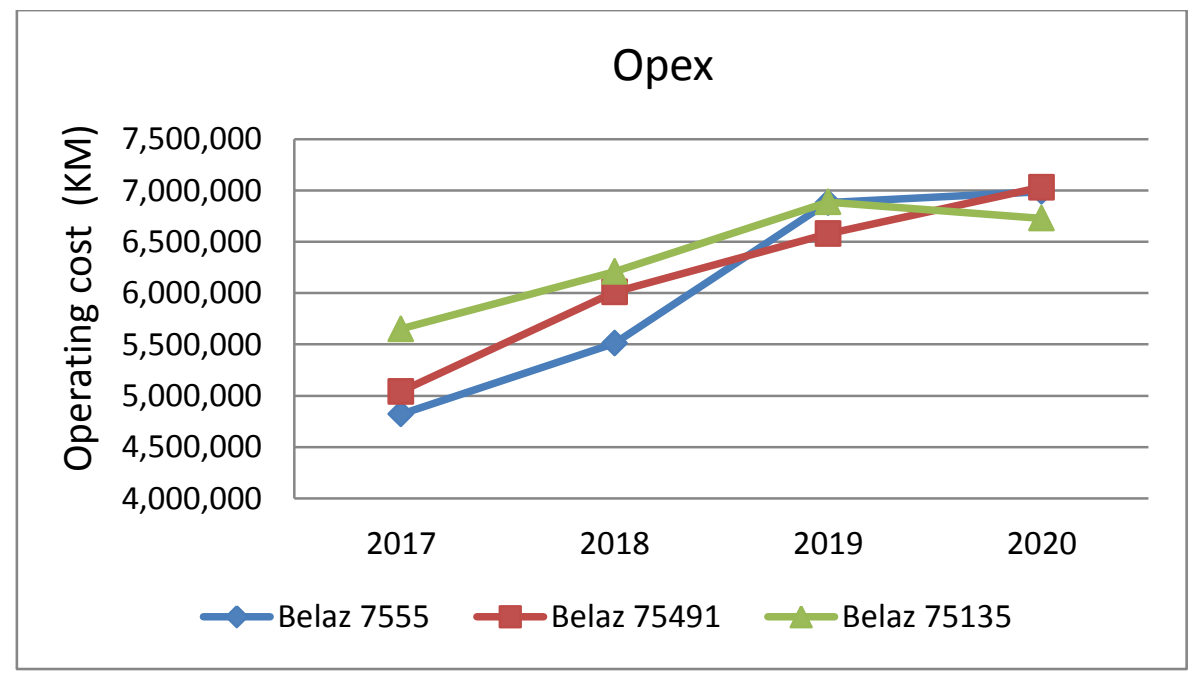

Figure 3 Operating costs by type of truck 


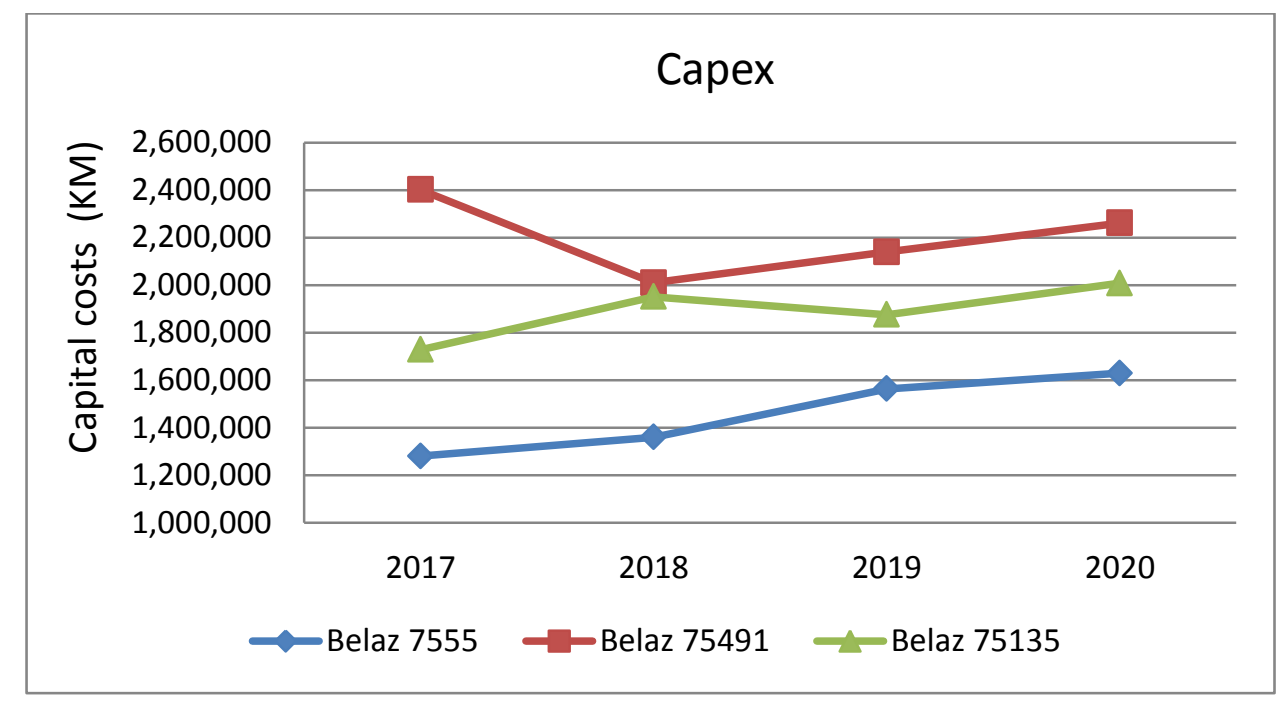

Figure 4 Capital costs by type of truck

\section{TRANSPORT COSTS PER VARIANT} SOLUTIONS

Specific costs of transport according to and the values are shown in the diagram in the variant embodiments given as a ratio of Fi-gure 5. Cost unit quantity of material is the total cost and weight needed to conduct provided in the $\mathrm{HP} / \mathrm{m}^{3}$. necessary transported per year periods,

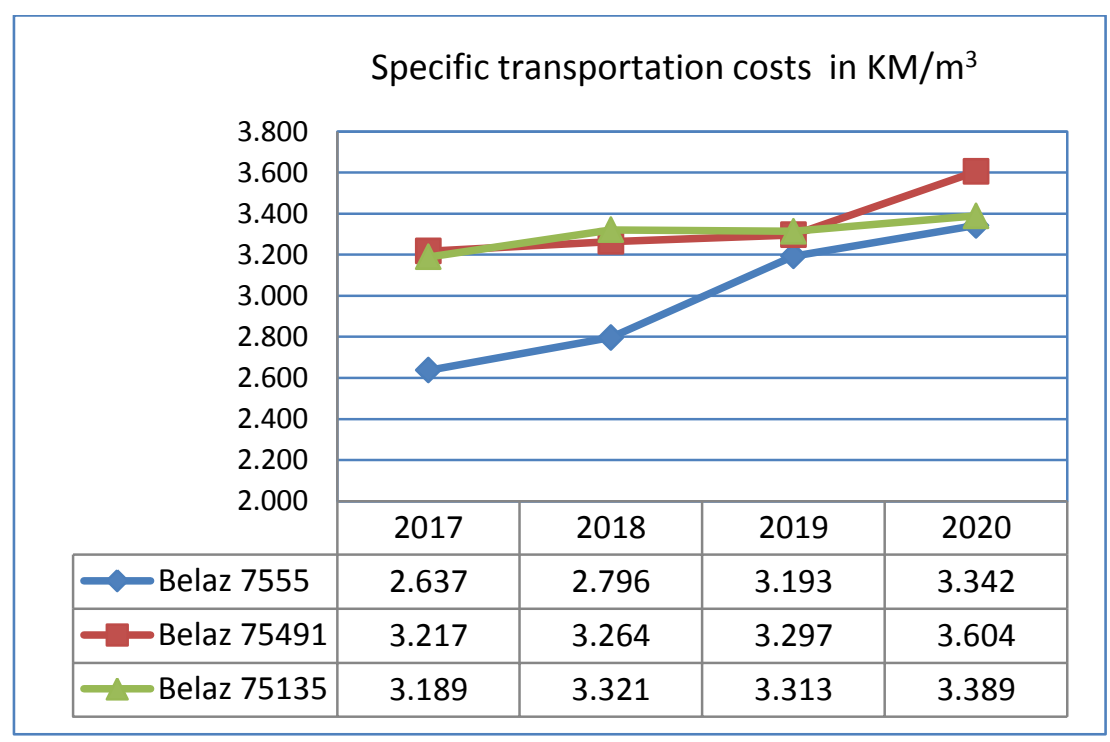

Figure 5 Specific transportation costs $\mathrm{KM} / \mathrm{m}^{3}$ 
Figure 6 shows the specific transport costs, expressed per meter of transport. These specific costs are given for the medium annual transport lengths. A medium transport length per some years is calculated as a weighted mean of length of transport route by the amount of material that is transported.

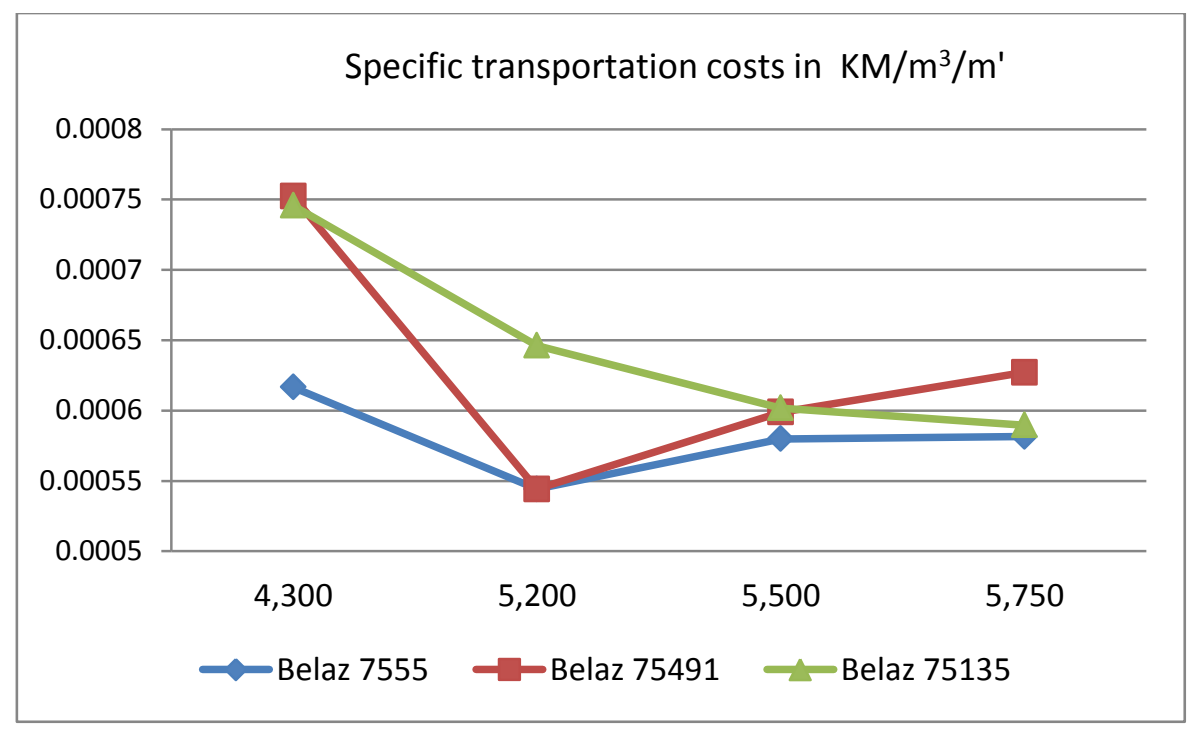

Figure 6 Costs per meter of transport route

\section{CONCLUSION}

Based on the calculated values of the technical and economic parameters of transport, as the most type of truck is allocated a truck Belaz 7555 capacity $55 \mathrm{t}$. The optimal type of truck can be determined through the present procedure of analysis, which has to satisfy the relevant require-ments.

The analysis was required on:

- specific ratio the amount of coal and seam waste in the deposit and defined level of selection in exploitation;

- specific physical and mechanical properties of coal and seam waste;

- $\quad$ specific transport lengths of coal and seam waste;

- specific site within the open pit, if there are more.
All listed parameters significantly affect the efficiency of operation the specific truck model, and a good knowledge of physical mechanical and structural characteristics of the work environment and technological characteristics of excavation equipment must be to make the final assessment on the best model of vehicle. When considering different models of trucks in addition to the technical operating parameters, such as the hourly capacity, capacity utilization factor, time utilization, etc. must be analyzed as well as the economic parameters of material transport for a specific model of truck that would cover both operating and capital costs.

In this case, the most favorable model truck is a truck Belaz 7555, which has the 


\section{REFERENCES}

smallest capacity and the volume of the crate. This conclusion can be drawn only for the period under consideration, or for consideration the weighted average duration of transport. The presented analysis shows that the increase of transport length, the parameters on the basis of which the asse-ssment of the transportation economy change in favor of the vehicles with a higher load-carrying capacity and volume of crates. The limit of application in relation to the other model of vehicle, in this particular case is the model 7555 Belaz up to 6 kilometers, while in the longer distances better para-meters can be expected in the application a truck Belaz 75135 .

The question of selecting the optimal type of truck in selective exploitation must be solved through a complex analysis for each specific case. This derives from the specificity of transport in selective exploittation considering that the same type of truck is used for transport of waste and transport of useful minerals. Also, the analysis must include a longer period of time in order to take into account the changeable conditions of exploitation in the real space of the open pit and real-time of exploitation.
[1] Strategy of Mining-Technological Opening, Development, Optimization And Maintenance of The Continuity of Production of The Coal With Introduction The Process of Coal Enrichment in The Dry Separation OP Gacko; MMI Bor, 2015 (in Serbian)

[2] The Main Design of The Open Pit Mining Gacko - Central Field For The Capacity of $2.3 \times 10^{6}$ T/A of Run-ofMine Coal, The Defense Project of The Open Pit Defense Against Water, MMI Bor D.O.O. Zvornik, Zvornik, 2016 (in Serbian)

[3] Tutorial - Talpac 9.0 Truck Loader \& Productivity Analysis Software, Tutorial - Metric, Runge Limited, Brisbane, Australia, 2007.

[4] Truck Transport at The Open Pit Mines, University of Belgrade, Belgrade RGF, Ranko Borovic, 1995 (in Serbian)

[5] Calculation of transport Means, University of Belgrade, Belgrade RGF, Ranko Borovic, 1987 (in Serbian) 